\title{
Épidémiologie des amibes libres dans les eaux de Strasbourg
}

\author{
par C. DERR-HARF *, B. MOLET **, J. SCHREIBER *** et M. KREMER ** \\ * Laboratoire de Parasitologie (Pr A. Ledoux), Faculté de Pharmacie, F 67000 Strasbourg. \\ * Institut de Parasitologie de la Faculté de Médecine de Strasbourg (Dir.: Pr M. Kremer), \\ 3, rue Koeberlé, F 67000 Strasbourg. \\ **a Laboratoire d'Hydrologie (Directeur: Pr J. Schreiber), Faculté de Pharmacie, \\ F 67000 Strasbourg.
}

\section{Résumé.}

Nous avons isolé 164 souches d'amibes libres des eaux des réseaux de distribution, des piscines et des baignades de Strasbourg; 11 genres et 16 espèces ont été identifiés. Certaines souches d'Acanthamoeba sont pathogènes pour la souris par inoculation intra-cérébrale. Aucune des deux souches de Naegleria isolées n'est pathogène. Nous comparons les résultats des recherches d'amibes libres avec les taux de chlore actif et de brome ainsi que la présence de bactéries dans les piscines couvertes.

\section{Summary.}

Epidemiology of free-living amoebae in the waters of Strasbourg (France).

164 strains of free-living amoebae were isolated from public drinking water supplies, swimming pools and official swimming ponds in Strasbourg; 11 genera and 16 species were identified. Some strains of Acanthamoeba are pathogenic for mice by intracerebral inoculation. Among the two strains of Naegleria found none is pathogenic. The results concerning free-living amoebae are compared with the level of chlorine and bromine and with the presence of bacteria in swimming pools.

Accepté le 12 avril 1977. 


\section{Introduction}

L'objectif de ce travail est de rechercher les amibes libres dans les eaux des réseaux de distribution, des piscines et des baignades de Strasbourg afin de les identifier et de surveiller l'éventuelle présence d'espèces pathogènes. Nous avons également étudié les conditions de stérilisation existant dans les piscines afin de vérifier si les normes de la législation établies pour l'élimination des bactéries sont efficaces contre les amibes libres. Ce travail a fait l'objet d'une thèse (5).

\section{Matériel et méthode}

Les méthodes employées sont les mêmes que celles décrites par Molet et Kremer (12) ainsi que dans les résultats préliminaires de cette étude (10).

Rappelons cependant certains points :

- Les prélèvements nous sont fournis par le laboratoire d'Hydrologie de la Faculté de Pharmacie de Strasbourg qui contrôle les eaux du point de vue bactériologique, chimique et physique. Les échantillons sont recueillis stérilement dans des bouteilles de verre d'un litre préalablement stérilisées, puis apportées au laboratoire de Parasitologie de la Faculté de Médecine où ils sont traités comme suit: l'eau est filtrée sur une membrane d'acétate de cellulose de porosité de 1,2 $\mu \mathrm{m}$. Le filtre est déposé à l'envers sur une couche de gélose à $2 \%$ d'Agar coulée dans une boîte de Pétri et recouverte d'Aerobacter aerogenes vivants (14). L'incubation est faite à $37^{\circ}$, afin de sélectionner les souches d'amibes éventuellement pathogènes. Les amibes isolées sont identifiées par leurs caractères morphologiques observés en culture sur lame (24). Des tests de flagellation sont faits dans de l'eau distillée et de l' «Amoeba saline (13).

- Les souches de Naegleria et d'Acanthamoeba sont mises en culture respectivement dans les milieux CGVS et CGV (28).

- La pathogénicité éventuelle des souches de Naegleria et Acanthamoeba est testée sur souris blanche par inoculation intra-cérébrale $(2,8)$. Les cerveaux de souris atteintes de méningo-encéphalite amibienne primitive sont prélevés. L'examen direct et la mise en culture de petites parties du cerveau, ainsi que l'examen de coupes histologiques colorées à l'hémalun-éosine confirment la pathogénicité de la souche isolée.

- Toutes les manipulations s'effectuent stérilement dans un laboratoire réservé à l'étude des amibes libres. 


\section{Résultats}

\section{a) Répartition des espèces selon les prélèvements.}

Nous avons étudié 270 échantillons prélevés pendant une année, du mois de mars 1975 au mois de mars 1976. 135 échantillons contenaient des amibes libres, soit $50 \%$ (tableau I). Nous avons isolé des amibes libres dans tous les biotopes étudiés. En outre, les prélèvements d'eau de piscine contenaient assez fréquemment des nématodes et des flagellés et parfois des acariens. La positivité des prélèvements est croissante des puits et stations de pompage au réseau d'alimentation et aux piscines, c'est-àdire de la «source» au consommateur. On peut donc supposer qu'il y a prolifération d'amibes dans les canalisations.

Tableau I. - Origine et résultats des prélèvements.

\begin{tabular}{|c|c|c|c|c|c|}
\hline & & $\begin{array}{c}\text { Prélèvements } \\
\text { positifs }\end{array}$ & $\begin{array}{l}\text { Prélèvements } \\
\text { négatifs }\end{array}$ & Total & $\%$ de positivité \\
\hline \multicolumn{2}{|c|}{ 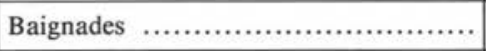 } & 6 & 0 & 6 & $100 \%$ \\
\hline \multicolumn{2}{|c|}{ Piscines en plein air $\ldots \ldots \ldots \ldots \ldots \ldots \ldots \ldots$} & 8 & 3 & 11 & $72,7 \%$ \\
\hline \multirow{3}{*}{$\begin{array}{l}\text { Piscines } \\
\text { couvertes } \\
\text { chauffées }\end{array}$} & Piscine A ........... & 37 & 23 & 60 & $61,7 \%$ \\
\hline & Piscine B ............ & 25 & 9 & 34 & $73,5 \%$ \\
\hline & Piscine $\mathrm{C} \ldots \ldots \ldots$ & 21 & 7 & 28 & $75 \%$ \\
\hline \multicolumn{2}{|c|}{ Réseau des hospices civils...$\ldots \ldots \ldots \ldots$} & 9 & 13 & 22 & $40,9 \%$ \\
\hline \multicolumn{2}{|c|}{ 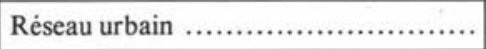 } & 17 & 31 & 48 & $35,4 \%$ \\
\hline \multicolumn{2}{|c|}{ Puits et stations de pompage $\ldots \ldots \ldots \ldots \ldots$} & 12 & 49 & 61 & $19,7 \%$ \\
\hline \multicolumn{2}{|c|}{ 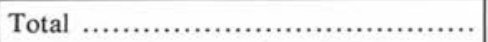 } & 135 & 135 & 270 & $50 \%$ \\
\hline
\end{tabular}

- Nous notons la présence plus fréquente d'amibes libres dans les échantillons d'eau de piscine que dans l'eau prélevée au robinet des piscines. La majorité des amibes libres isolées des bassins ne provient donc pas de l'eau du réseau qui les alimente. Une source de contamination pourrait provenir des baigneurs et de la poussière contenant des kystes d'amibes (26).

- Dans les piscines couvertes, la positivité des prélèvements varie selon les bassins et selon que le prélèvement a été effectué à l'entrée ou à la sortie du bassin (tableau II). On observe une plus forte positivité dans le grand bassin que dans le petit bassin pour les piscines $\mathrm{A}$ et $\mathrm{C}$, et le contraire pour la piscine $\mathrm{B}$.

L'eau contient plus fréquemment des amibes libres à son entrée dans les bassins qu'à sa sortie. Ceci est particulièrement net dans le grand bassin de la piscine $\mathrm{B}$, où 
Tableau II. - Résultats des piscines couvertes selon l'endroit du prélèvement

Piscine A

\begin{tabular}{|c|c|c|c|c|c|}
\hline & & $\begin{array}{c}\text { Prélèvements } \\
\text { positifs }\end{array}$ & $\begin{array}{c}\text { Prélèvements } \\
\text { négatifs }\end{array}$ & $\begin{array}{c}\% \text { de } \\
\text { positivité }\end{array}$ & $\begin{array}{l}\text { \% de positivité } \\
\text { du bassin }\end{array}$ \\
\hline \multirow{2}{*}{ Grand bassin } & Eftrée...$\ldots \ldots \ldots \ldots$ & 10 & 0 & $100 \%$ & \multirow{2}{*}{$95 \%$} \\
\hline & Sortie .. & 9 & 1 & $90 \%$ & \\
\hline \multirow{2}{*}{ Petit bassin ........ } & Entrée .............. & 8 & 2 & $80 \%$ & \multirow{2}{*}{$65 \%$} \\
\hline & Sortie .. & 5 & 5 & $50 \%$ & \\
\hline \multirow{2}{*}{ Bassin plongeoir } & Entrée ............... & 3 & 7 & $30 \%$ & \multirow{2}{*}{$25 \%$} \\
\hline & Sortie $\ldots \ldots \ldots \ldots \ldots$ & 2 & 8 & $20 \%$ & \\
\hline
\end{tabular}

Piscine B

\begin{tabular}{|c|c|c|c|c|c|}
\hline & & \begin{tabular}{c|}
$\begin{array}{c}\text { Prélèvements } \\
\text { positifs }\end{array}$ \\
\end{tabular} & \begin{tabular}{|c|}
$\begin{array}{c}\text { Prélèvements } \\
\text { négatifs }\end{array}$ \\
\end{tabular} & $\begin{array}{c}\% \text { de } \\
\text { positivité }\end{array}$ & $\begin{array}{l}\text { \% de positivité } \\
\text { du bassin }\end{array}$ \\
\hline \multirow{2}{*}{ Grand bassin } & Entrée .............. & 9 & 0 & $100 \%$ & \multirow{2}{*}{$72,2 \%$} \\
\hline & Sortie ... & 4 & 5 & $44,4 \%$ & \\
\hline \multirow{2}{*}{ Petit bassin } & Entrée. & 6 & 2 & $75 \%$ & \multirow{2}{*}{$75 \%$} \\
\hline & Sortie $\ldots \ldots \ldots \ldots . . . .$. & 6 & 2 & $75 \%$ & \\
\hline
\end{tabular}

Piscine C

\begin{tabular}{|c|c|c|c|c|c|}
\hline & & $\begin{array}{c}\text { Prélèvements } \\
\text { positifs }\end{array}$ & $\begin{array}{c}\text { Prélèvements } \\
\text { négatifs }\end{array}$ & $\begin{array}{c}\% \text { de } \\
\text { positivité }\end{array}$ & $\begin{array}{c}\% \text { de positivité } \\
\text { du bassin }\end{array}$ \\
\hline \multirow{2}{*}{ Grand bassin } & Entrée ............... & 7 & 0 & $100 \%$ & \multirow{2}{*}{$86,7 \%$} \\
\hline & Sortie . & 5 & 2 & $71 \%$ & \\
\hline \multirow{2}{*}{ Petit bassin } & Entrée .............. & 5 & 2 & $71 \%$ & \multirow{2}{*}{$64,3 \%$} \\
\hline & Sortie & 4 & 3 & $57 \%$ & \\
\hline
\end{tabular}

nous trouvons $100 \%$ de positivité à l'entrée et $44,4 \%$ à la sortie. Nous supposons qu'il y a une prolifération d'amibes dans les filtres, ce qui expliquerait leur fréquence à l'entrée. La multiplication d'amibes dans les filtres a été notée par d'autres auteurs : Cerva (1) dans la piscine d'Usti et Jadin (8) qui qualifie les filtres de «nids d'amibes ».

- Parmi les 135 prélèvements positifs, 21 contiennent 2 espèces et 4 contiennent 3 espèces d'amibes libres. Nous avons isolé ainsi 164 souches. Nous ne les avons volontairement pas clônées dans le but de tester leur pathogénicité à partir de souches hétérogènes. Nous avons résumé dans le tableau III, l'identification et la répartition des souches isolées. Nous avons déterminé 11 genres et 16 espèces parmi les 131 souches; 33 souches n'ont pas pu être identifiées. 
Tableau III. - Identification et répartition des souches isolées

\begin{tabular}{|c|c|c|c|c|c|c|c|c|c|c|}
\hline & \multicolumn{3}{|c|}{$\begin{array}{l}\text { Piscines } \\
\text { couvertes } \\
\text { chauffées }\end{array}$} & \multicolumn{2}{|c|}{$\begin{array}{l}\text { Piscines } \\
\text { plein air }\end{array}$} & \multirow[t]{2}{*}{ Baign. } & \multirow{2}{*}{$\begin{array}{c}\text { Réseau } \\
\text { des } \\
\text { hospices } \\
\text { civils }\end{array}$} & \multirow{2}{*}{$\begin{array}{c}\text { Réseau } \\
\text { urbain }\end{array}$} & \multirow{2}{*}{$\begin{array}{c}\text { Puits et } \\
\text { stations } \\
\text { de pom- } \\
\text { page }\end{array}$} & \multirow[t]{2}{*}{ Total } \\
\hline & A & B & $\mathrm{C}$ & $\mathrm{D}$ & $\mathrm{E}$ & & & & & \\
\hline Acanthamoeba polyphaga.......... & 8 & 1 & 5 & 1 & 1 & 2 & - & - & 3 & 21 \\
\hline Acanthamoeba castellanii ............ & 2 & 5 & 3 & - & 1 & - & - & - & 1 & 12 \\
\hline Acanthamoeba comandoni $\quad . . . . . . . .$. & - & 1 & - & - & - & - & - & - & - & 1 \\
\hline Acanthamoeba lenticulata ........... & - & - & 1 & - & - & - & - & - & 1 & 2 \\
\hline Hartamannella vermiformis ........... & 20 & 3 & 5 & 2 & 2 & 3 & 3 & 4 & 5 & 47 \\
\hline Saccamoeba limax .................. & - & 1 & - & - & - & - & - & - & - & 1 \\
\hline Paratetramitus jugosus ................ & - & - & - & - & - & 2 & - & - & - & 2 \\
\hline Naegleria spp ........................... & - & - & - & - & - & 1 & 1 & - & - & 2 \\
\hline Platyamoeba stenopodia...$\ldots \ldots \ldots \ldots$ & 3 & 1 & - & - & - & - & 1 & 3 & - & 8 \\
\hline Vannella platypodia $. . . \ldots \ldots \ldots \ldots \ldots . . . . .$. & - & - & - & - & - & - & - & - & 2 & 2 \\
\hline Vannella mira...$\ldots \ldots \ldots \ldots \ldots \ldots \ldots$ & 3 & 1 & 5 & - & 1 & - & 1 & 1 & - & 12 \\
\hline Rosculus ithacus .................... & - & 8 & 2 & - & - & - & - & 1 & - & 11 \\
\hline Vexillifera bacillipedes ................ & 3 & 1 & 1 & - & - & - & - & - & 5 & \\
\hline Echinamoeba exundans ............... & 1 & - & - & - & - & - & - & - & - & 1 \\
\hline Echinamoeba silvestris .............. & - & - & 3 & - & - & - & - & - & - & 3 \\
\hline Filamoeba nolandi..$\ldots \ldots \ldots \ldots \ldots \ldots$ & - & - & - & 1 & - & - & - & - & - & 1 \\
\hline Petites amibes $6 \mu$ m sans kystes $\ldots . .$. & 1 & 1 & - & - & - & - & 2 & 4 & - & 8 \\
\hline Amibes pâles $11 \mu \mathrm{m}$ sans kystes ..... & - & 1 & - & - & - & - & - & 3 & - & 4 \\
\hline Souches indéterminées ............... & 3 & 7 & 1 & - & 3 & - & 1 & 6 & - & 21 \\
\hline Total & 44 & 31 & 26 & 4 & 8 & 8 & 9 & 22 & 12 & 164 \\
\hline
\end{tabular}

- Acanthamoeba polyphaga (Puschkarew, 1913) emend. Page, $1967 b$ (14)

- Acanthamoeba castellanii (Douglas, 1930) emend. Volkonsky, 1931

- Acanthamoeba comandoni Pussard, $1964 b$ (23)

- Acanthamoeba lenticulata Molet et Ermolieff-Braun, 1976 (II)

- Hartmannella vermiformis Page, $1967 a$ (13)

- Saccamoeba limax (Dujardin, 1841) emend. Page, 1969, (16)

- Paratetramitus jugosus Darbyshire, Page et Goodfellow, 1976 (3)

- Naegleria spp Alexeieff 1912, emend. Calkins 1913

- Platyamoeba stenopodia Page, 1969* (18)

- Vannella platypodia (Glaser, 1912) emend. Page 1976 (21)

- Vannella mira (Schaeffer, 1926) emend. Page 1976 (21)

- Rosculus ithacus Hawes, 1963, emend. Page, 1974 (19)

- Vexillifera bacillipedes Page, 1969* (17)

- Echinamoeba exundans Page, $1975^{*}(20)$

- Echinamoeba silvestris Page, $1975^{*}(20)$

- Filamoeba nolandi Page, $1967 c^{*}(15)$

* Remarques : nos observations concernant la mitose de ces espèces diffèrent de celles de Page (5) Les particularités des mitoses des genres Platyamoeba et Echinamoeba feront l'objet d'une publication ultérieure. 
Nous constatons une nette prédominance des genres Hartmannella (47 souches) et Acanthamoeba (36 souches). La répartition des populations d'amibes est différente selon les biotopes :

- Hartmannella vermiformis est présente dans tous les biotopes.

- Le genre Acanthamoeba est fréquent dans les piscines, on le trouve également dans les puits et les stations de pompage, mais il est absent des réseaux.

- Platyamoeba stenopodia, Vannella mira et Rosculus ithacus sont présents dans les réseaux et dans les piscines.

- Les genres Vexillifera et Echinamoeba ne se rencontrent que dans les piscines.

Deux souches seulement sont du genre Naegleria.

Elles ont été isolées l'une dans une baignade et l'autre dans le réseau des Hospices civils. Nous n'en avons pas trouvé dans les piscines.

L'une des souches est probablement $N$. gruberi (Schardinger, 1899) l'autre est proche de $N$. jadini, Willaert et Le Ray, 1973 (29). Nous n'avons pas isolé de $N$. fowleri, Carter, 1970.

\section{b) Pathogénicité des amibes isolées.}

Nous avons testé la pathogénicité des Naegleria et des Acanthamoeba. En effet, c'est pour ces deux genres que des affections humaines ont été décrites.

L'inoculation intra-cérébrale nécessite l'emploi de formes végétatives en culture axénique. Seule la souche de Naegleria proche de $N$. jadini s'est bien développée en milieu CGVS à $37^{\circ}$. En outre, neuf souches d'Acanthamoeba ont pu être axénisées en milieu $\mathrm{CGV}$ à $28^{\circ}$.

Les résultats des inoculations sont rassemblés dans le tableau IV. La souche de Naegleria a été inoculée à 4 lots de souris. Toutes les souris ont survécu pendant plus de 2 mois. Cette souche ne semble donc pas être pathogène. Par contre, une souche d' $A$. polyphaga, les trois souches $d$ 'A. castellanii, la souche d'A. lenticulata et celle d'A. comandoni sont pathogènes pour la souris.

A notre connaissance, ceci est la première démonstration de la pathogénicité d'A. comandoni.

Nous avons retrouvé des formes végétatives dans des coupes de cerveaux de souris inoculées avec des Acanthamoeba pathogènes. La présence de kystes n'a pas été mise en évidence.

\section{c) Fréquence des amibes et taux de chlore et de brome.}

Nous avons comparé les résultats des recherches d'amibes libres avec les taux de chlore actif et de brome ainsi que la présence de bactéries dans les piscines couvertes.

La piscine A est traitée par le brome, les piscines B et C le sont par le chlore.

La législation requiert un taux minimum de brome de $0,4 \mathrm{mg} / 1$. Pour le chlore, le taux de chlore actif, c'est-à-dire d'acide hypochloreux doit être compris entre 0,2 et $0,5 \mathrm{mg} / 1$ à la sortie de chaque bassin. On détermine la teneur en chlore libre 


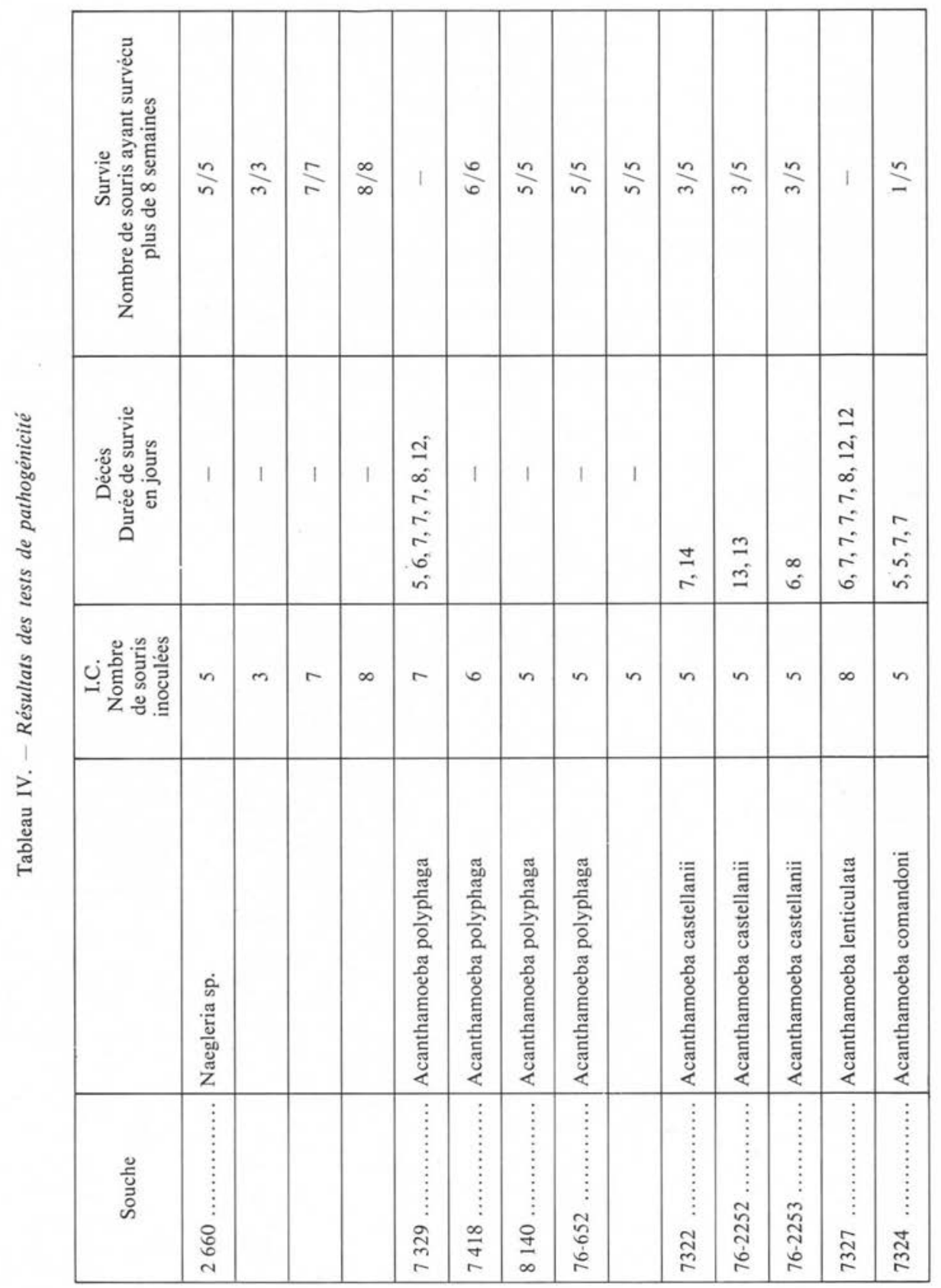


mesurée par la méthode de Palin au diéthyl-paraphénylène-diamine (22) en tenant compte du pH. Dans une étude sur l'action du chlore sur les amibes de l'eau, Derreumaux et coll. (6) démontrent que l'efficacité de la stérilisation sur les amibes est directement fonction de la teneur en chlore actif. Ils préconisent un taux minimum de $0,5 \mathrm{mg} / 1$ de chlore actif. Nous avons consigné dans les tableaux V et VI la fréquence d'isolement d'amibes libres par rapport à la teneur en chlore actif des échantillons des piscines B et C. Dans la piscine B, nous constatons que pour les taux inférieurs à $0,20 \mathrm{mg} / 1$ et ceux supérieurs à $0,50 \mathrm{mg} / 1$ de chlore actif, on trouve la même fréquence de positivité en amibes libres $(60 \%)$. Dans la piscine $\mathrm{C}$, les prélèvements ayant une teneur en chlore actif supérieure à $0,50 \mathrm{mg} / 1$ contiennent plus souvent des amibes libres $(100 \%)$ que ceux situés en-dessous de ce taux $(72 \%)$. Ces résultats ne nous permettent pas d'établir une relation entre la teneur en chlore actif des piscines et la présence d'amibes libres. Cette absence de corrélation confirme l'opinion d'autres auteurs comme Cerva (1), de Jonckheere (4) et Desmet-Paix (7). L'action du brome sur les amibes libres a été peu étudiée jusqu'à présent. Nous comparons dans le tableau VII, la fréquence d'isolement d'amibes libres avec la teneur en brome dans les prélèvements de la piscine A. Ici aussi, il n'est pas possible d'établir de corrélation. Le pourcentage de positivité en amibes libres élevé pour les taux de brome inférieurs à $0,9 \mathrm{mg} / 1$ s'abaisse entre 0,9 et $1,2 \mathrm{mg} / \mathrm{l}$.

Tableaux V et VI. - Comparaison des résultats de recherche d'amibes libres avec la teneur en chlore actif des piscines $B$ et $C$.

Tableau V. Piscine B

\begin{tabular}{|c|c|c|c|}
\hline Chlore actif en mg/1 & Nombre d'échantillons & $\begin{array}{c}\text { Nombre d'échantillons } \\
\text { contenant } \\
\text { des amibes libres }\end{array}$ & $\begin{array}{l}\% \text { de positivité } \\
\text { en amibes libres }\end{array}$ \\
\hline $0,10-0,19 \ldots \ldots \ldots \ldots \ldots \ldots \ldots$ & 5 & 3 & $60 \%$ \\
\hline 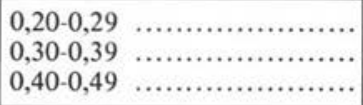 & $\left.\begin{array}{r}13 \\
6 \\
5\end{array}\right\} 24$ & $\left.\begin{array}{r}11 \\
6 \\
2\end{array}\right\} 19$ & $79 \%$ \\
\hline 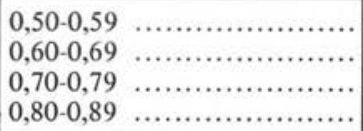 & $\left.\begin{array}{l}1 \\
2 \\
2\end{array}\right\} 5$ & $\left.\begin{array}{l}1 \\
1 \\
1\end{array}\right\} 3$ & $60 \%$ \\
\hline
\end{tabular}

Tableau VI. - Piscine C

\begin{tabular}{|c|c|c|c|}
\hline Chlore actif en mg/l & Nombre d'échantillons & $\begin{array}{c}\text { Nombre d'échantillons } \\
\text { contenant } \\
\text { des amibes libres }\end{array}$ & $\begin{array}{l}\text { \% de positivité } \\
\text { en amibes libres }\end{array}$ \\
\hline 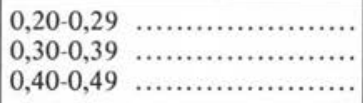 & $\left.\begin{array}{r}8 \\
10 \\
7\end{array}\right\} 25$ & $\left.\begin{array}{l}5 \\
8 \\
5\end{array}\right\} 18$ & $72 \%$ \\
\hline 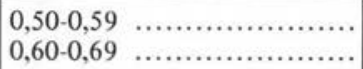 & $\left.\begin{array}{l}2 \\
1\end{array}\right\} 3$ & $\left.\begin{array}{l}2 \\
1\end{array}\right\} 3$ & $100 \%$ \\
\hline
\end{tabular}



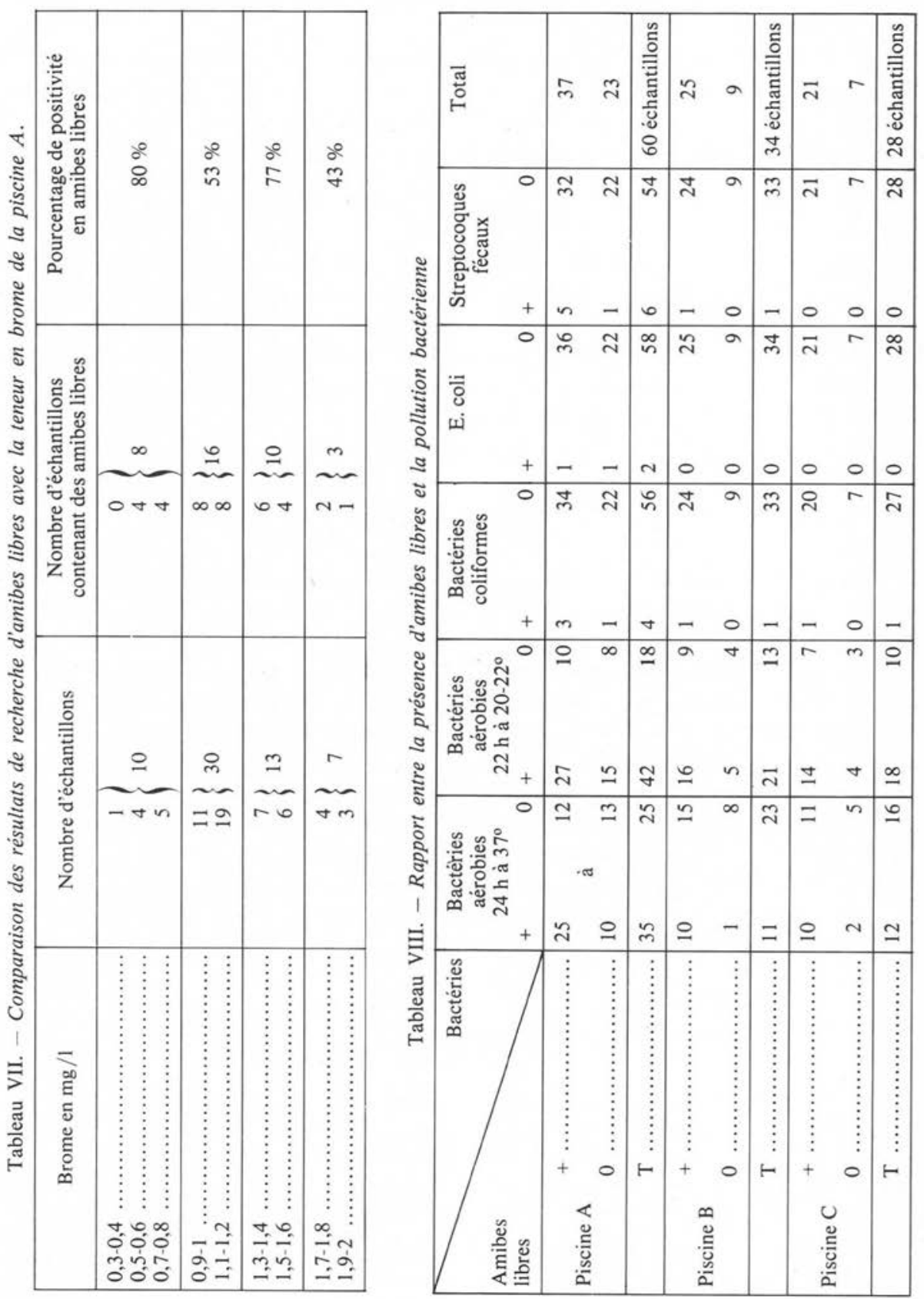
Mais il remontre entre 1,3 et $1,6 \mathrm{mg} / 1$ pour diminuer à nouveau pour les taux élevés de brome de 1,7 à $2 \mathrm{mg} / 1$.

Ainsi la stérilisation des piscines par le chlore et par le brome ne semble pas être efficace sur les amibes libres.

\section{d) Présence d'amibes et flore microbienne associée.}

Nous comparons la présence d'amibes et celle de bactéries dans les piscines couvertes (tableau VIII).

Ces statistiques ne laissent pas apparaître l'aspect quantitatif de la recherche mais seulement la présence ou l'absence des amibes et des germes recherchés. Dans la plupart des prélèvements contenant des bactéries on trouve aussi des amibes libres. En outre, très peu d'échantillons contiennent des amibes libres en l'absence de bactéries. Ceci nous semble logique, les amibes libres étant tributaires de la présence de bactéries dont elles se nourrissent essentiellement.

\section{Conclusion}

La présence en abondance d'amibes libres, dont certaines du genre Acanthamoeba sont pathogènes, représente un danger potentiel pour les utilisateurs d'eau du réseau et des piscines.

Il ressort de cette étude que les taux de chlore et de brome requis par la législation sont insuffisants pour éliminer les amibes libres. Même à forte dose, ces agents stérilisants semblent n'avoir qu'une action très limitée. Des recherches approfondies s'avèrent nécessaires afin d'adapter les normes actuelles à la lutte contre les amibes.

Ii semble que les amibes prolifèrent dans les filtres des piscines. Nous avons entrepris une étude sur ce problème. Dès à présent, nous pouvons recommander l'application de mesures telles que le nettoyage quotidien des filtres à $70^{\circ}$ (1), et la préchloration (27) ainsi que l'élimination des agglomérats de matières organiques (8).

Ces mesures préventives permettraient de diminuer la prolifération amibienne et de réduire le danger potentiel qu'elle implique.

\section{Bibliographie}

1. Cerva L. (1971): Studies of limax amoebae in a swimming pool. Hydrobiologia, 38, 141-161.

2. Culbertson C. G., Smith J. W., Cohen H. K. et Minner J. R. (1959): Experimental infection of mice and monkeys by Acanthamoeba. Am. J. Clin. Pathol., 35, 185-197.

3. Darbyshire J. F., Page F. C. et Goodfellow L.P. (1976): Paratetramitus jugosus and amoeboflagellate of soils and freshwater, type-species of Paratetramitus nov. gen. Protistologica, 12, 375-387.

4. De Jonckheere J. et Van de Voorde H. (1976): Differences in destruction of cysts of pathogenic and non pathogenic Naegleria and Acanthamoeba by chlorine. Appl. Envir. Microbiol., 31, 294-297. 
5. Derr-Harf C. (1977): Etude des amibes libres dans les eaux de Strasbourg. Thèse Doct. Pharmacie, Strasbourg, $141 \mathrm{p}$.

6. Derreumaux A.L., Jadin J.B., Willaert E. et Moret R. (1974): Action du chlore sur les amibes de l'eau. Proc. Intern. Coll. Antwerp., 191-204.

7. Desmet-Paix L. et Fisch-Thiébaut C. (1975): La présence d'amibes dans les piscines couvertes. Note d'orientation. Labo. Intercommunal Chimie et Bact., Bruxelles, 18 p.

8. Jadin J.B. (1974): De la méningo-encéphalite amibienne primitive et du pouvoir pathogène des amibes de l'eau. Bull. Acad. Med. Belg., 129, 439-466.

9. Martinez A. J., Nelson E. C. et Duma R. J. (1973): Animal model : primary amebic (Naegleria) meningo-encephalitis in mice. Am. J. Pathol., 73, 545-548.

10. Molet B., Derr-Harf C., Schreiber J. et Kremer M. (1976): Etude des amibes libres dans les eaux de Strasbourg. Résultats préliminaires. Ann. Parasitol. Hum. Comp., 51, 401-406.

11. Molet B. et Ermolieff-Braun G. (1976): Description d'une amibe d'eau douce Acanthamoeba lenticulata sp. nov. (Amoebida). Protistologica, 12, 571-576.

12. Molet B. et Kremer M. (1976): Techniques d'étude et critères morphologiques pour l'identification des amibes libres. Bull. Soc. Sci. Vet. Med. Comp., (Lyon), 78, 215-224.

13. Page F.C. $(1967 a)$ : Taxonomic criteria for limax amoebae, with description of 3 new species of Hartmannella and 3 of Vahlkampfia. J. Protozool., 14, 709-713.

14. Page F.C. $(1967 b)$ : Redefinition of the genus Acanthamoeba with description of three species. J. Protozool., 14, 709-713.

15. Page F. C. $(1967$ c) : Filamoeba nolandi n. gen., n. sp., a filose amoeba. Trans. Am. Micr. Soc., $86,405-411$.

16. Page F.C. (1969): Hartmannella limax: the original limax amoeba? Trans. Am. Micr. Soc., 88, 199-204.

17. Page F.C. (1969): Mitosis and pseudopod formation in Vexillifera bacillipedes n. sp., a mayorellid amoeba. Trans. Am. Micr. Soc., 88, 394-400.

18. Page F. C. (1969): Platyamoeba stenopodia n. g., n. sp., a fresh-water amoeba. J. Protozool., $16,437-441$.

19. Page F.C. (1974): Rosculus ithacus Hawes 1963 (Amoebida, Flabellulidae) and the amphizoic tendancy in amoeba. Acta Protozool., 13, 143-154.

20. Page F.C. (1975): A new family of amoebae with fine pseudopodia. Zool. J. Linn. Soc., 56, 73-89.

21. Page F. C. (1976): An illustrated key to freshwater and soil amoebae with notes on cultivation and ecology. Freshwater Biol., Ass. Sc. Publ., n ${ }^{\circ} 34$.

22. Palin (1957): The determination of free and combined chlorine in water by the use of NN diethyl p.phénylene diamine. J. Am. W. W. Ass., 49, 873-880.

23. Pussard M. (1964 b): Acanthamoeba comandoni n. sp. Comparaison avec A. Terricola Pussard. Rev. Ecol. Biol. Sol. 1, 587-610.

24. Pussard M. (1974): La morphologie des amibes libres, intérêt et principes d'études. Ann. Soc. Belge Med. Trop., 54, 249-257.

25. Singh B. N. (1950): A culture method for growing small free-living amoebae for the study of their nuclear division. Nature, 165, 65-66.

26. Skocil V., Cerva L. et Serbus C. (1971 a) : Epidemiological study of amoebae of the limax group in military communities. Relation between the findings of amoebae in the external environment and their incidence in the soldiers during the investigation into the community L-IV. J. Hyg., Epid., Microbiol., Immunol., 15, 445-449.

27. White G. C. (1972): Handbook of chlorination. Van Nostrand Reinhold, édit., New York.

28. Willaert E. (1971): Isolement et culture in vitro des amibes du genre Naegleria. Ann. Soc. Belge Med. Trop., 51, 701-708.

29. Willaert E. et Le Ray D. (1973): Caractères morphologiques, biologiques et immunochimiques de Naegleria jadini, sp. nov. (Amoebida, Vahlkampfiidae). Protistologica, 9, 417-426. 